\title{
MicroRNA-375 targets PDK1 in pancreatic carcinoma and suppresses cell growth through the Akt signaling pathway
}

\author{
JIAN ZHOU ${ }^{1 *}$, SHIDUO SONG $^{1 *}$, SONGBING HE $^{1,2^{*}}$, XINGUO ZHU $^{1 *}$, YI ZHANG $^{1}$, \\ ${\text { BIN } \text { YI }^{1}, \text { BING ZHANG }}^{3}$, GONGZHAO QIN $^{4}$ and DECHUN LI ${ }^{1}$ \\ ${ }^{1}$ Department of General Surgery, The First Affiliated Hospital of Soochow University, Suzhou, Jiangsu 215006; \\ ${ }^{2}$ Shanghai Institute of Immunology, Shanghai Jiao Tong University School of Medicine, Shanghai 200025; \\ ${ }^{3}$ Department of Nuclear Medicine, The First Affiliated Hospital of Soochow University, Suzhou, Jiangsu 215006; \\ ${ }^{4}$ Department of Gynaecology and Obstetrics, Suzhou Municipal Hospital, Suzhou, Jiangsu 215003, P.R. China
}

Received October 17, 2013; Accepted January 24, 2014

DOI: $10.3892 / \mathrm{ijmm} .2014 .1638$

\begin{abstract}
MicroRNAs (miRNAs or miRs) are believed to have great potential for use as molecular targets in the diagnosis and treatment of cancer. In this study, we demonstrate that miR-375 is downregulated in pancreatic carcinoma (PC) tissues and PC cell lines. We found that miR-375 negatively regulates the expression of 3-phosphoinositide-dependent protein kinase 1 (PDK1) by directly targeting the 3'UTR of the PDK1 transcript. To investigate the biological roles and the potential mechanisms of action of miR-375, we induced either the up- or downregulation of miR-375 expression by transfecting various PC cells with miR-375 mimics or an inhibitor. Our results revealed that the upregulation of miR-375 inhibited cell growth and induced cell apoptosis, while the downregulation of miR-375 with the inhibitor had the opposite effect. In addition, our data demonstrate that miR-375 suppresses the malignant behavior of PC cells through the Akt signaling pathway rather than mitogen-activated protein kinase (MAPK) signaling pathways. Taken together, our findings indicate that targeting miR-375 by a genetic approach may provide a novel strategy for the treatment of PC.
\end{abstract}

\section{Introduction}

Pancreatic carcinoma (PC) is one of the most lethal and aggressive of all malignancies. It is the sixth leading cause

Correspondence to: Dr Dechun Li, Department of General Surgery, The First Affiliated Hospital of Soochow University, 188 Shizi Street, Suzhou, Jiangsu 215006, P.R. China

E-mail: zhoujian0612@gmail.com

${ }^{*}$ Contributed equally

Key words: microRNA-375, 3-phosphoinositide-dependent protein kinase 1, pancreatic carcinoma, Akt of mortality from malignant disease in China and the fourth leading cause of cancer-related mortality in the United States $(1,2)$. The median overall survival is $>6$ months and the 5-year survival rate is dismal, ranging between 2 and 6\% (3). Despite advances in chemotherapy and radiotherapy over the past few decades, the overall prognosis of PC has remained essentially unchanged (4). Recently, the classical categories of oncogenes and tumor suppressor genes have been expanded to include a novel class of small non-coding RNAs, termed microRNAs (miRNAs or miRs), which can regulate a number of protein-coding genes, including tumor-related genes (5).

miRNAs regulate genes expression by base pairing with partially complementary messenger RNAs (mRNAs), resulting in the degradation of target mRNAs or the inhibition of their translation. The expression patterns of miRNAs appear to be tissue-specific and more accurate than mRNAbased profiling in tumor diagnostics. To date, a number of deregulated miRNAs have been found in human malignancies, including breast cancer, lung cancer, hepatocellular carcinoma (HCC) and colon cancer (6-8). Among these miRNAs, miR-375 was originally reported to be an islet cellspecific miRNA and to regulate insulin secretion (9). Recent studies have demonstrated the downregulation of miR-375 in gastric cancer (10), HCC (11) and colorectal cancer (12). However, the function and mechanisms of action of miR-375 in PC have not yet been fully elucidated.

To determine the function of miR-375 as a tumor suppressor gene, we searched for physiological targets using TargetScan (http://www.targetscan.org/) and found that 3-phosphoinositide-dependent protein kinase 1 (PDK1) is a putative target of miR-375. As a pleckstrin homology $(\mathrm{PH})$ domain-containing protein, PDK1 has emerged as an important oncogene in multiple types of cancer (13). It has been reported that PDK1 is overexpressed in human PC and promotes cancer cell proliferation, growth and invasion (14). However, knowledge of the mechanisms that regulate PDK1 expression during tumor progression is limited.

In this study, we investigated the biological roles and the potential mechanisms of action of miR-375 in PC. Furthermore, we identified PDK1 as a putative target of miR-375 by compu- 
tational prediction and luciferase reporter assays. Our findings demonstrated that miR-375 may have great potential for use as molecular targets in the diagnosis and treatment of PC.

\section{Materials and methods}

Clinical samples. Tissue samples from 50 patients with PC were collected during surgical resections performed at the First Affiliated Hospital of Soochow University, Suzhou, China from January 2010 to December 2012. Tumor tissues and adjacent non-tumor tissues were frozen immediately after surgical removal in liquid nitrogen and stored at $-80^{\circ} \mathrm{C}$. The patients had not received any pre-operative chemotherapy, radiotherapy or immunotherapy. All samples were obtained after receiving patient consent from and approval from the Ethics Committee of Soochow University.

Cell culture and transfection. The human PC cell lines, PANC-1, SW1990, Capan-1, Patu8988, AsPC-1 and BxPC-3 obtained from the Shanghai Institute of Cell Biology (Shanghai, China) were maintained in DMEM or RPMI-1640 supplemented with 10\% fetal bovine serum (all from Gibco, Grand Island, $\mathrm{NY}, \mathrm{USA}$ ) and $100 \mu \mathrm{g} / \mathrm{ml}$ each of penicillin and streptomycin (Invirtrogen, Carlsbad, CA, USA) in $5 \% \mathrm{CO}_{2}$ at $37^{\circ} \mathrm{C}$.

Cells $\left(1 \times 10^{4}\right)$ per well were cultured in 6-well plates overnight until they reached $60-70 \%$ confluence. Due to their different expression levels of miR-375 (PANC-1 cells express low levels of miR-375 and BxPC-3 cells express high levels) the PANC-1 and BxPC- 3 cells were selected and transfected with miR-375 mimics (mimics group), miR-375 inhibitor (inhibitor group) or a negative control (NC group) (GeneChem, Shanghai, China) using Lipofectamine 2000 (Invitrogen, Carslbad, CA, USA) as the transfection reagent. Non-transfected cells were used as the blank control (mock group).

Quantitative reverse transcription polymerase chain reaction $(q R T-P C R)$. Total RNA was extracted the from tissues and cells using TRIzol reagent (Invitrogen). The expression levels of miR-375 were measured using a TaqMan microRNA assay (Applied Biosystems, Foster City, CA, USA) specific for hsa-miR-375 in the PCR reactions of 40 cycles $\left(95^{\circ} \mathrm{C}, 15 \mathrm{sec}\right.$; $60^{\circ} \mathrm{C}, 60 \mathrm{sec} ; 70^{\circ} \mathrm{C}, 30 \mathrm{sec}$ ), with U6 RNA as an internal control. PDK1 expression was detected with the following primers: forward, 5'-GTGTAGATTAGAGGGATG-3' and reverse, 5'-AAGGAATAGTGGGTTAG G-3' and $\beta$-actin was used as an internal control with the following primers: forward, 5 'AGC GAGCATCCCCCAAAGTT-3' and reverse, 5'-GGGCACGAA GGCTCATCATT-3'. The PCR reactions consisted of 40 cycles $\left(95^{\circ} \mathrm{C}, 15 \mathrm{sec} ; 62^{\circ} \mathrm{C}, 45 \mathrm{sec} ; 72^{\circ} \mathrm{C}, 30 \mathrm{sec}\right)$. The amplified segments were analyzed on $2.5 \%$ agarose gels.

Western blot analysis. The cells were collected and lysed in lysis buffer on ice. Total proteins were separated by $10 \%$ SDS-PAGE and blotted onto PVDF membranes. The membranes were then blocked with $10 \%$ non-fat milk powder at room temperature for $2 \mathrm{~h}$ and incubated with the following primary antibodies: anti-PDK1 antibody (1:100), anti-GAPDH antibody (1:300) (both from Santa Cruz Biotechnology, Inc., Santa Cruz, CA, USA), anti-extracellular signal-regulated kinase (ERK)1/2 antibody (1:100), anti-Akt antibody (1:100), anti-p38 antibody (1:100) (all from CST Technologies, Inc., Chicago, IL, USA) at $4^{\circ} \mathrm{C}$ overnight. After 3 washes, the membranes were incubated with a horseradish peroxidaseconjugated goat anti-mouse IgG (1:2,000; Santa Cruz Biotechnology, Inc.) for $2 \mathrm{~h}$ at room temperature. Reactive bands were detected using ECL Western Blotting Detection Reagent (GE Healthcare, Pittsburgh, PA, USA).

Luciferase reporter assay. To verify that PDK1 is a direct target of miR-375, the wild-type 3'UTR (WT 3'UTR) of PDK1 containing the miR-375 binding site was amplified using the following primers: forward, 5'-ACCCAACCACACAAA GAACAAAA-3' and reverse, 5'-TTTTGTTCTTTGTGTGG TTGGGT-3'. As a negative control, the mutated binding site of the 3'UTR (MUT 3'UTR) was amplified using the following primers: forward, 5'-ACCCAACCACACAAAGACACAAA-3' and reverse, 5'-TTTACAAGATTGTGTGGTTGGGT-3'. Products were cloned into the luciferase reporter vector, pMIR-Report (Ambion, Austin, TX, USA). PANC-1 cells were seeded in 24-well plates and co-transfected with the luciferase reporter vector and miR-375 mimics or miR-negative control (NC) as described above. The cells were harvested $48 \mathrm{~h}$ after transfection and luciferase activity was measured using the Dual-Luciferase assay system (Promega, Madison, WI, USA).

Cell proliferation assay. The CCK8 assay was performed to determine cell growth. Following transfection, the cells were seeded in 96 -well plates at $5 \times 10^{3}$ cells per well, followed by the addition of $20 \mu \mathrm{l}$ CCK8 (Dojindo Laboratories, Kumamoto, Japan) and incubation at $37^{\circ} \mathrm{C}$ for an additional $2 \mathrm{~h}$. An ultraviolet spectrophotometer was used to measure the absorbance of each well at $450 \mathrm{~nm}$, and each experiment was performed in triplicate and repeated 3 times.

Analysis of cell apoptosis . After $72 \mathrm{~h}$ of transfection, the cells were seeded and a volume of $100 \mu \mathrm{l}$ of cell suspension $\left(1 \times 10^{6}\right.$ cells $\left./ \mathrm{ml}\right)$ was labeled with $10 \mu \mathrm{l}$ of propidium iodided (PI) and $5 \mu \mathrm{l}$ of Annexin V/FITC (both from BD Biosciences, Franklin Lakes, NJ, USA). The cells were incubated in the dark for $15 \mathrm{~min}$ at room temperature and the number of early apoptotic cells was determined using a FACSCalibur flow cytometer (BD Biosciences).

Terminal dUTP nick end-labeling (TUNEL) assays. The cells were plated on glass coverslips in 24-well plates and fixed in $4 \%$ paraformaldehyde for $60 \mathrm{~min}$. After washing with phosphate-buffered saline, $50 \mu \mathrm{l} /$ well Cy3-labeling TUNEL (Beyotime, Shanghai, China) reaction mixture were added to the cells followed by incubation for $60 \mathrm{~min}$ at $37^{\circ} \mathrm{C}$ in the dark. The TUNEL-positive cells emitted red fluorescence under a fluorescence microscope. The number of apoptotic cells was calculated by counting the number of positive cells in at least 5 randomly selected microscopic fields (magnification, $\mathrm{x} 200$ ).

Statistical analysis. SPSS software version 17.0 was used for statistical analysis. Data re expressed as the means \pm standard deviation (SD). One-way analysis of variance (one-way Anova) or the t-test was performed for inter-group comparisons. Pearson's correlation analysis was used to compare 

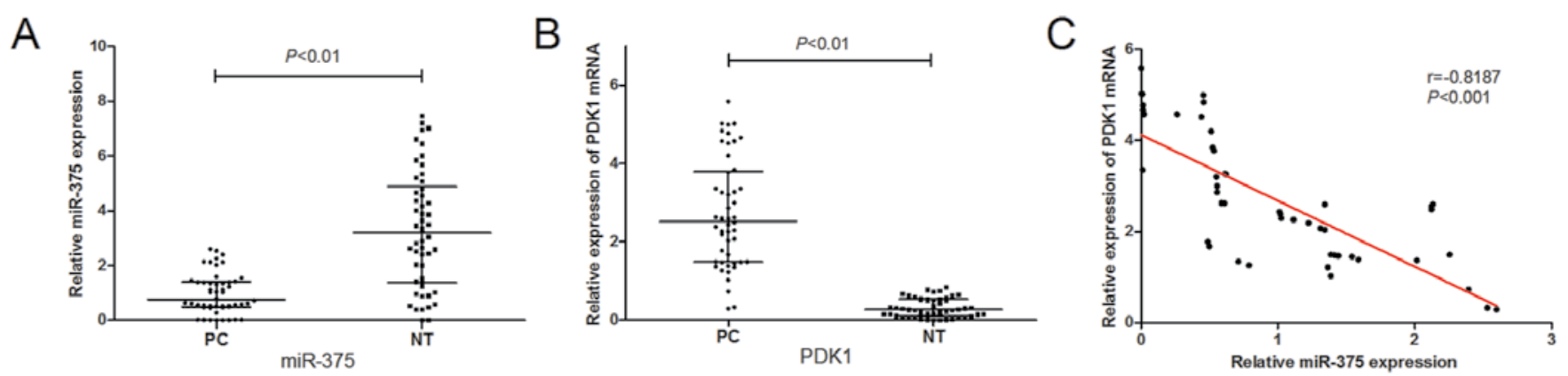

Figure 1. Inverse correlation between the expression of microRNA-375 (miR-375) and 3-phosphoinositide-dependent protein kinase 1 (PDK1) in pancreatic carcinoma (PC). (A) The expression of miR-375 was examined in PC tissues and non-tumor tissues (NT) by qRT-PCR. (B) The expression of PDK1 mRNA was examined in PC and NT tissues by qRT-PCR. (C) miR-375 inversely correlated with PDK1 mRNA expression (P<0.001, Pearson's correlation).

A

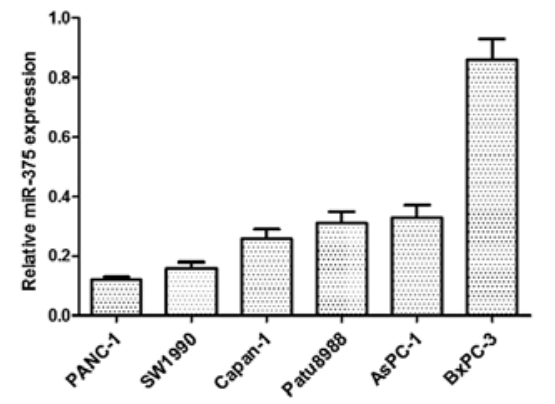

B

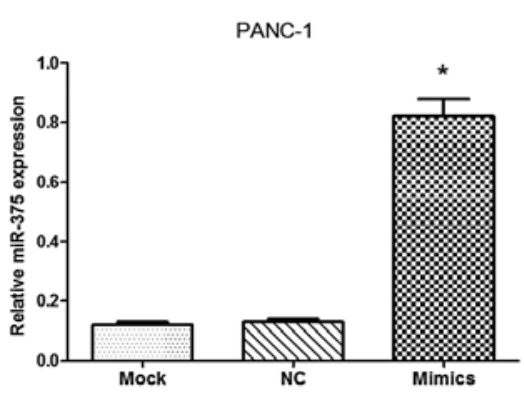

C

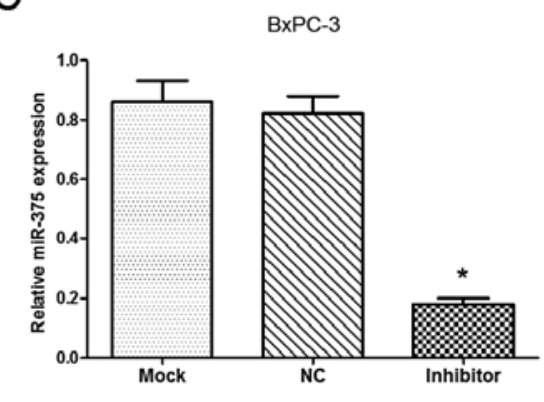

Figure 2. microRNA-375 (miR-375) mimics and inhibitor transfection in pancreatic carcinoma (PC) cells. (A) Relative expression of miR-375 in 6 PC cell lines (PANC-1, SW1990, Capan-1, Patu8988, AsPC-1 and BxPC-3). (B) miR-375 expression following transfection with miR-375 mimics or negative control (NC) in PANC-1 cells assessed by qRT-PCR ( $\mathrm{P}<0.05)$. (C) miR-375 expression following transfection with miR-375 inhibitor or NC in BxPC-3 cells assessed by qRT-PCR $\left({ }^{*} \mathrm{P}<0.05\right)$.

miR-375 expression with PDK1 expression. A value of $\mathrm{P}<0.05$ was considered to indicate a statistically significant difference.

\section{Results}

miR-375 is downregulated and inversely correlates with PDK1 in PC. To determine the expression levels of miR-375 in PC, we examined miR-375 expression in 50 pairs of PC tissues and matched adjacent non-tumor tissues by qRT-PCR. As shown in Fig. 1A, the miR-375 expression levels were significantly suppressed in the $\mathrm{PC}$ tissues $(\mathrm{P}<0.01)$. Of note, the expression of miR-375 inversely correlated with that of PDK1, a potential target of miR-375 (Fig. 1B and C).

miR-375 is a post-transcriptional regulator of PDK1. We examined the relative expression of miR-375 in $6 \mathrm{PC}$ cell lines (PANC-1, SW1990, Capan-1, Patu8988, AsPC-1 and BxPC-3), from which the PANC-1 and BxPC-3 cells were selected, as they were found to have a low and high expression of miR-375, respectively (Fig. 2A). As shown in Fig. 2B and C, the levels of miR-375 were significantly upregulated in the PANC-1 cells following transfection with miR-375 mimics $(\mathrm{P}<0.05)$, while the levels of miR-375 were signficantly downregulated in the BxPC-3 cells following transfection with the miR-375 inhibitor $(\mathrm{P}<0.05)$.
Furthermore, the expression of PDK1 was examined by qRT-PCR and western blot analysis in these 2 cell lines. Our data revealed no apparent change in PDK1 expression at the transcript level (Fig. 3A), while the PDK1 protein level was significantly downregulated in the cells transfected with miR-375 mimics and upregulated in the cells transfected with the miR-375 inhibitor compared with the NC-transfected cells (Fig. 3B; both $\mathrm{P}<0.05$ ).

PDK1 is a direct target of miR-375. To confirm the direct targeting of PDK1 by miR-375, we integrated a firefly luciferase reporter plasmid containing a region of PDK1 3'UTR harboring the miR-375 target site, or a fragment whose target site was mutated (Fig. 4A), and used these to co-transfect the PANC-1 cells with miR-375 mimics or miR-NC. As shown in Fig. 4B, our results demonstrated that relative luciferase activity was decreased in the WT 3'UTR-transfected PANC-1 cells that were co-transfected with miR-375 mimics as compared with those cells co-transfected with miR-NC $(\mathrm{P}<0.05)$, while the mutation of the miR-375 binding site blocked this suppressive effect. These results strongly suggest that miR-375 negatively regulates the expression of PDK1 by directly targeting the 3'UTR of the PDK1 transcript.

Effect of miR-375 on proliferation and apoptosis of PC cells. Using the transfected cells, CCK8 assays were performed 

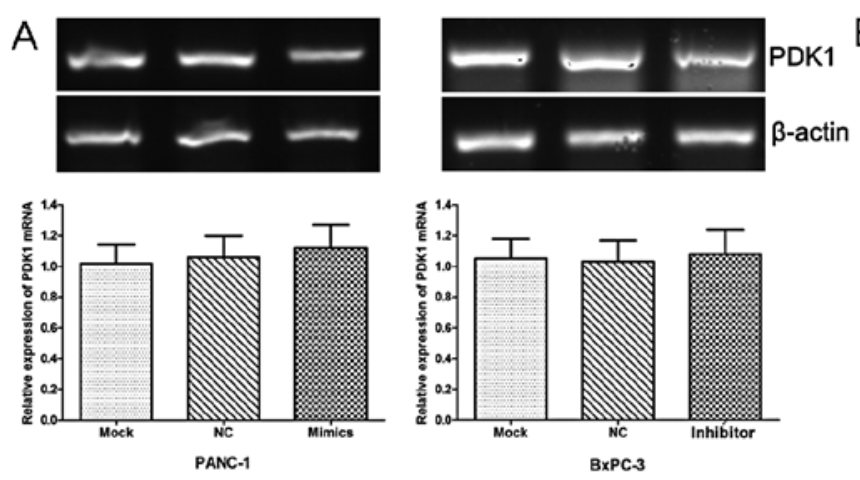
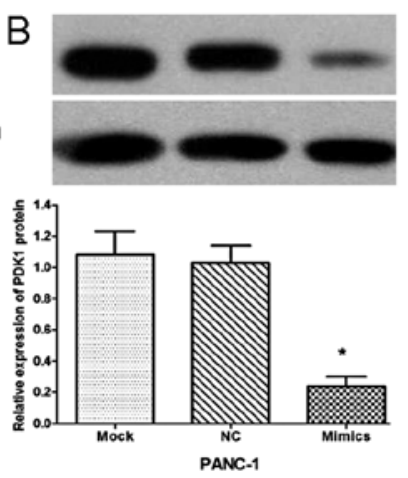

Figure 3. microRNA-375 (miR-375) negatively regulates the expression of 3-phosphoinositide-dependent protein kinase 1 (PDK1). (A) The expression of PDK1 mRNA was examined by qRT-PCR and amplified products were also run on a $1 \%$ agarose gel. (B) The expression of PDK1 protein was assessed by western blot analysis $(* \mathrm{P}<0.05)$.

A

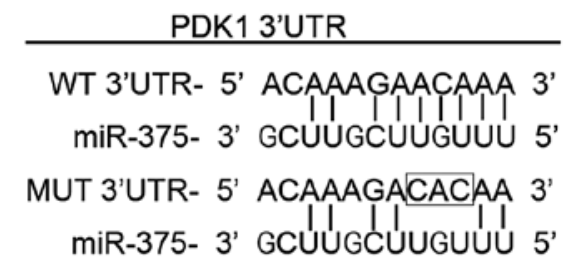

B

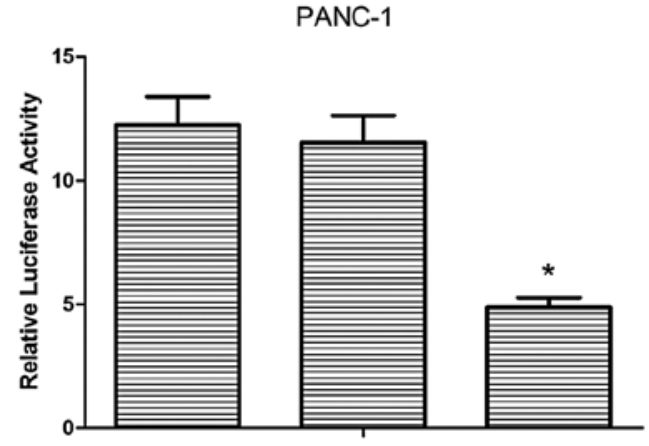

$\begin{array}{lccc}\text { WT 3'UTR } & + & - & + \\ \text { MUT 3'UTR } & - & + & - \\ \text { MiR-NC } & + & - & - \\ \text { miR-375 } & - & + & +\end{array}$

Figure 4. 3-Phosphoinositide-dependent protein kinase 1 (PDK1) is a direct target of microRNA-375 (miR-375). (A) Schematic representation of luciferase reporter construct containing PDK1 3'UTR with either the wild-type (WT) or mutant (MUT) miR-375 target site. (B) Luciferase reporter assay was carried out to examine the control of gene expression mediated by miR-375 $\left({ }^{*} \mathrm{P}<0.05\right)$.

to examine the effects of miR-375 on PC cell proliferation. As shown in Fig. 5A, the PANC-1 cells transfected with the miR-375 mimics displayed significant growth inhibition compared with the cells transfected with miR-NC at 48 and $72 \mathrm{~h}$ (both $\mathrm{P}<0.05$ ). On the contrary, the BxPC-3 cells transfected with the miR-375 inhibitor showed an enhanced proliferation at 48 and $72 \mathrm{~h}(\mathrm{P}<0.05)$.

We further assessed the effects of miR-375 on cell apoptosis by flow cytometry and TUNEL assays. The apoptotic rate in the PANC-1 cells transfected with the miR-375 mimics was significantly higher than that of the cells transfected with miR-NC (Fig. 5B; $\mathrm{P}<0.05$ ). In the BxPC-3 cells, the cells transfected with the miR-375 inhibitor showed a decreased rate of apoptosis compared with the miR-NC-transfected group $(\mathrm{P}<0.05)$. As shown in Fig. 5C, TUNEL assays demonstrated that the number of apoptotic cells in the miR-375 mimic-transfected group was greater than that in the miR-NCtransfected group (miR-mimic-transfected cells, 120.16 \pm 10.04 cells per field; miR-NC-transfected cells, 31.06 \pm 4.57 cells per field; $\mathrm{P}<0.05)$. The downregulation of miR-375 induced by the inhibitor led to a decrease in the number of apoptotic cells (miR-375 inhibitor-transfected cells, 42.56 7.11 cells per field; miR-NC-transfected cells, 131.01 \pm 12.32 cells per field; $\mathrm{P}<0.05)$.

Effect of miR-375 on mitogen-activated protein kinase (MAPK) and Akt signaling pathways in PC cells. PDK1 is an oncogene involved in the regulation of cell cycle progression, apoptosis and invasion through the MAPK or Akt signaling pathways. Thus, we investigated the effects of miR-375 on the activation status of MAPK and Akt pathways by western blot analysis (Fig. 6). Our results revealed the decreased expression of phosphorylated (p)-Akt in the miR-375 mimic-transfected cells and an increased expression of p-Akt in the miR-375 inhibitor-transfected group as compared with the controls (NC). There was no change observed in the expression of ERK1/2, p-ERK1/2, p38, p-p38 and Akt. These results suggest that miR-375 suppresses the malignant behavior of PC cells through the Akt signaling pathway.

\section{Discussion}

In the present study, we investigated the role of miR-375 in PC and observed a tendency towards the downregulation of miR-375 expression in PC tissues compared to normal (nontumor) ones. We induced either the up- or downregulation of miR-375 expression by transfecting PC cells with miR-375 mimics or miR-375 inhibitor and observed that miR-375 suppressed tumor cell proliferation and induced apoptosis. Furthermore, our results demonstrated that the 3'UTR of human PDK1 contained a putative target of miR-375 and that miR-375 regulated PDK1 expression at the protein level in PC cells.

miRNAs are small non-coding RNA molecules that play key roles in cell proliferation, apoptosis and differentiation in cancer initiation and progression (15). A great deal of evidence 
A

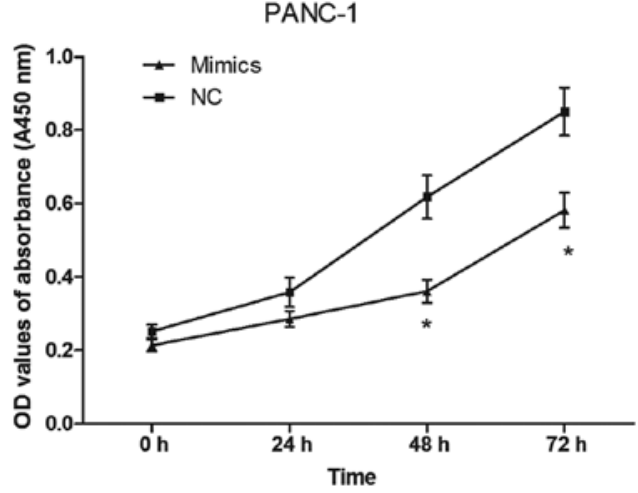

BxPC-3

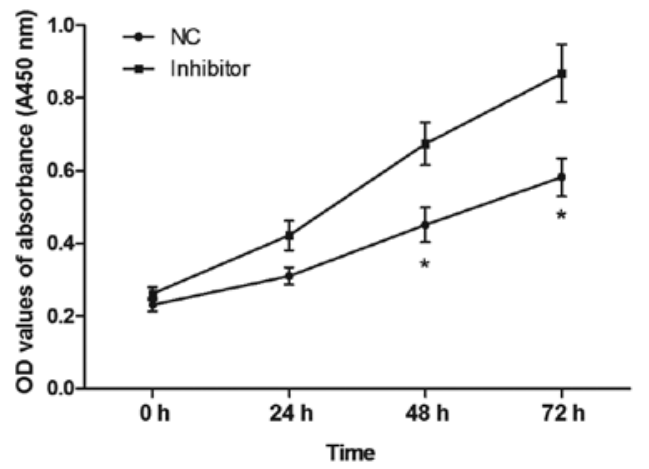

B
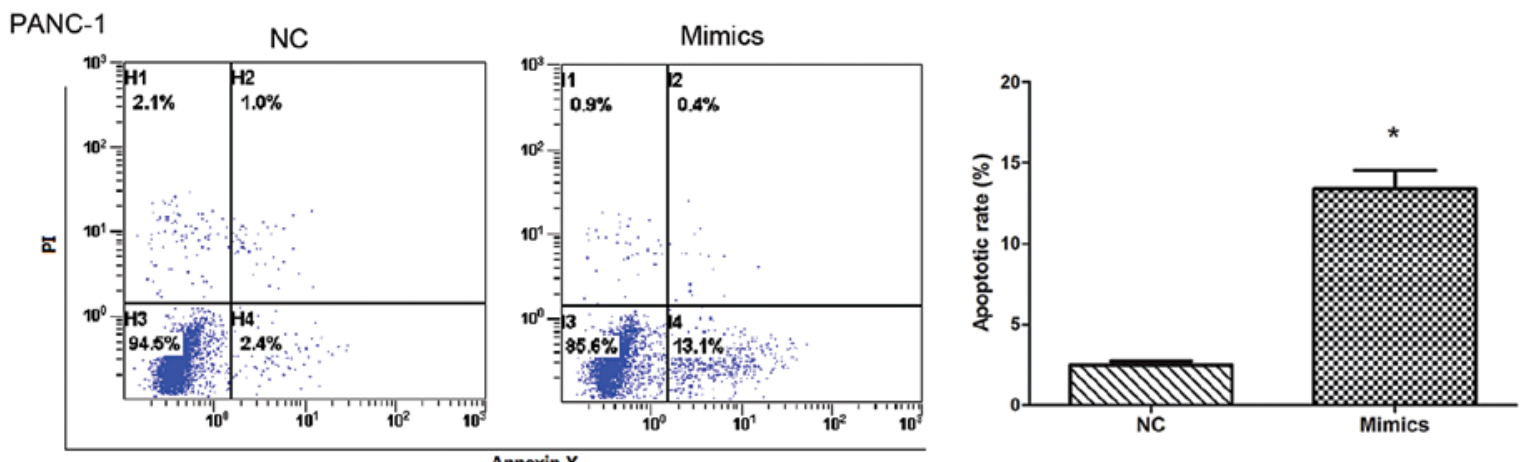

BxPC-3
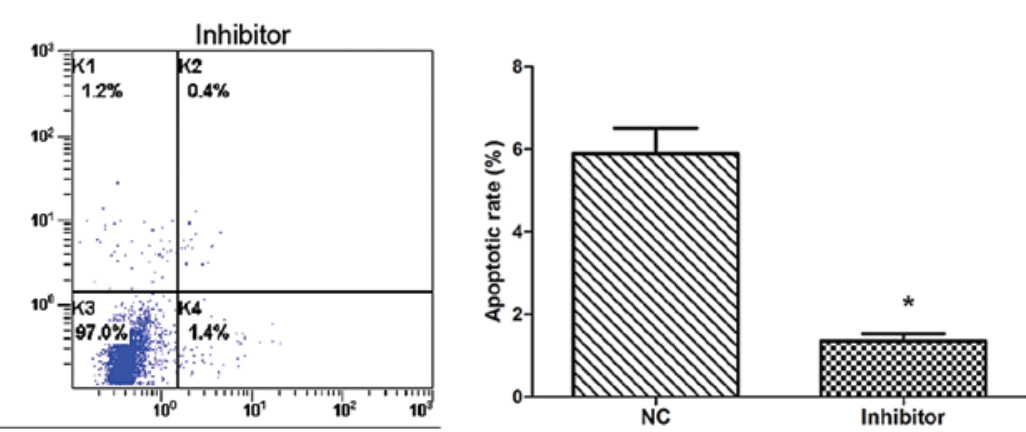

C
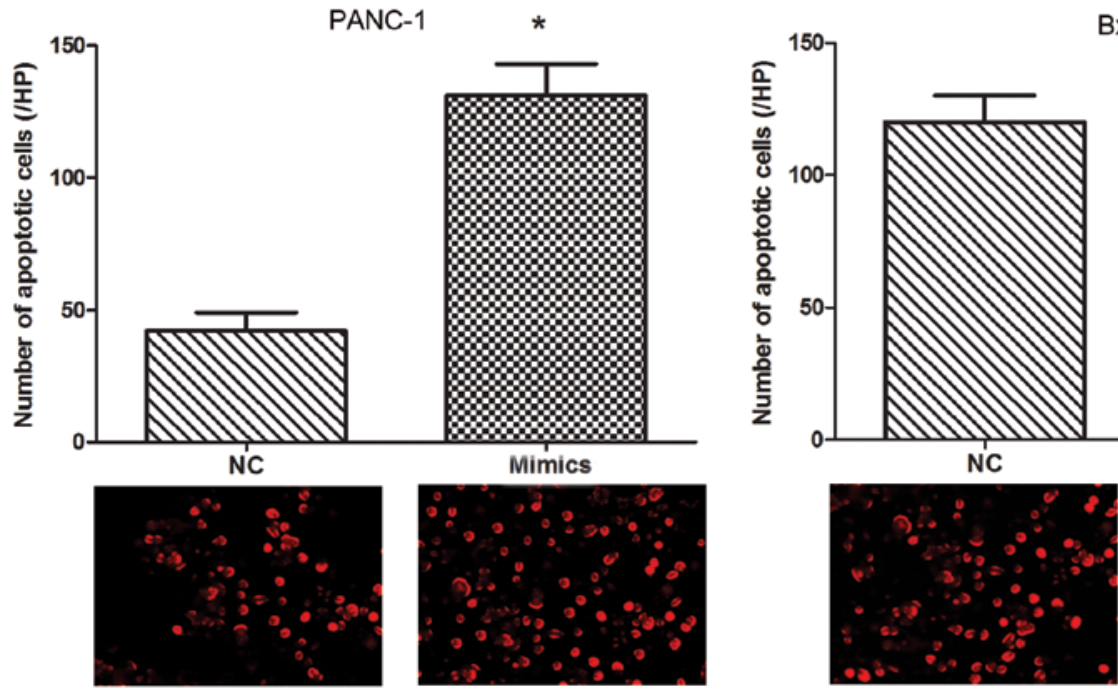

BxPC-3

Figure 5. Effects of microRNA-375 (miR-375) on cell growth and apoptosis. (A) Cell viability following transfection with miR-375 mimics or inhibitor was analyzed by CCK8 assay ("P<0.05). (B) Apoptotic cell rate following transfection with miR-375 mimics or inhibitor was detected by Annexin V/propidium iodide (PI) combined labeling flow cytometry ( $\left.{ }^{*} \mathrm{P}<0.05\right)$. (C) The number of apoptotic cells following transfection with miR-375 mimics or inhibitor in PANC-1 or BxPC-3 cells was counted under a fluorescence microscope by Cy3-labeling TUNEL assay (magnification, $\mathrm{x} 200$ ). 


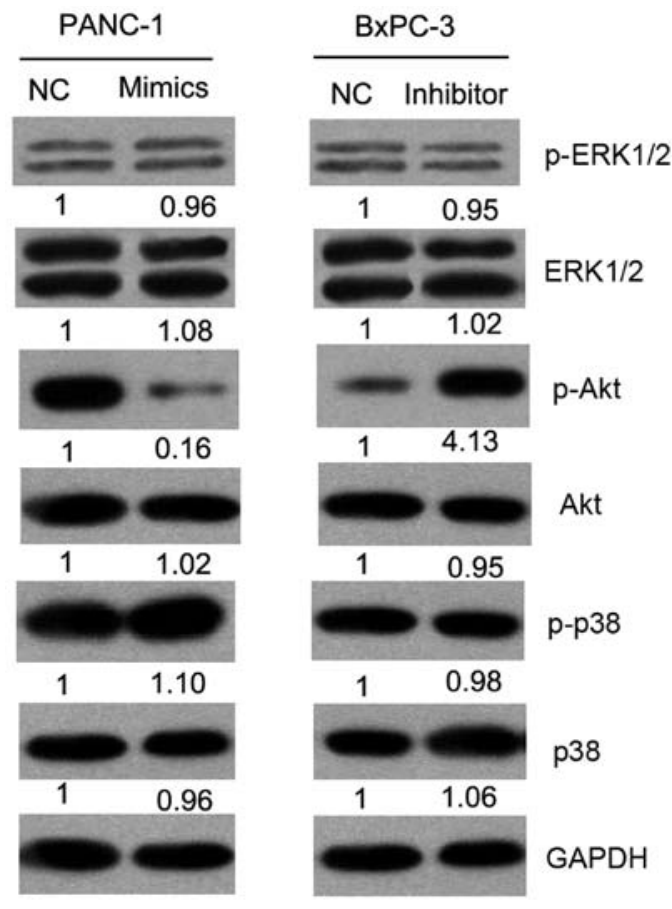

Figure 6. Impact of microRNA-375 (miR-375) on protein expression on MAPK and Akt signaling pathways. Western blot analysis was performed to determine the protein expression of ERK1/2, phosphorylated (p)-ERK1/2, Akt, pAkt, p38, pP38 and GAPDH (as an internal control) followed by densitometry of immunoreactive bands. Normalized densitometric values are indicated at the bottom of the bands.

has suggested that miRNAs act either as oncogenes or tumor suppressor genes in various types of cancer $(5,7)$. In this study, our results revealed that the upregulation of miR-375 in PC cells suppressed their malignant behavior and that the downregulation of miR-375 with the inhibitor had the opposite effect. These data suggest that miR-375 acts as a tumor suppressor gene in PC. Although miR-375 was initially shown to be expressed in pancreatic islets (9), it is worth mentioning that the aberrant expression of miR-375 has also been reported in other malignancies (10-12). In the majority of studies, miR-375 has been shown to suppress cell growth and proliferation, such as in HCC (16) and gastric cancer (17). However, the overexpression of miR-375 in cervical cancer cells has been shown to decrease sensitivity to paclitaxel (18). These data from other studies, together with our findings indicate that the function of miR-375 in cancer is cel type-specific.

miRNAs exert their their regulatory effects on gene expression through partial complementary elements in the 3'UTR of their target mRNA, resulting in transcript degradation or translational arrest (19). Since a single miRNA can target multitudinous genes, the important targets of miR-375 include yes-associated protein (YAP), PDK1 and Janus kinase 2 (JAK2) (20-22). These targets play diverse roles in different cellular systems. For instance, YAP1 is regulated by miR-375 and inhibits cell growth in lung cancer (23). miR-375 targets p53 in gastric cancer cells and abrogates cell cycle arrest and apoptosis (24). Our results demonstrated that miR-375 suppressed PDK1 expression by binding directly to the 3'UTR of PDK1 and an inverse correlation was observed between
miR-375 and PDK1 expression in the PC clinical specimens. PDK1 is a key component of the PI3K/Akt signaling pathway and its overexpression has been associated with cell growth and proliferation in PC. Thus, the identification of miR-375 as a regulator of PDK1 may have potential therapeutic implications in PC and other malignancies.

ERK1/2and p38, members of MAPKs, are important cellular protein kinases. Akt, also known as protein kinase B, is a serine/threonine protein kinase. The activation of Akt, ERK1/2 and p38 through phosphorylated forms plays a crucial role in the modulation of cell survival and apoptosis $(25,26)$. Previous studies have demonstrated that miR-375 is involved in regulating target genes in the MAPK signal pathway $(27,28)$. In this study, our data demonstrated that miR-375 modulated the phosphorylation of Akt in PC cells, while no change was observed in p-ERK1/2 and p-p38 expression. To the best of our knowledge, our observations that miR-375 effectively influenced PC cell proliferation and apoptosis through the Akt signaling pathway have not been previously reported. Therefore, our findings provide a more comprehensive understanding of miR-375 in the development and progression of PC.

In conclusion, this study demonstrates that miR-375 negatively regulates the oncogene, PDK1, and modulates PC cell proliferation and apoptosis through the Akt signaling pathway. Our findings indicate that targeting miR-375 through a genetic approach may provide a novel strategy for the treatment of PC.

\section{Acknowledgements}

This study was supported by grants from the National Natural Science Foundation of China (81201905), the China Postdoctoral Science Foundation(2013M540374), the Shanghai Postdoctoral Scientific Program of China (13R21415200) and Natural Science Research Grants from the University of Jiangsu Province of China (12KJB320009).

\section{References}

1. Chen X, Ma S and Zhang Z: Analysis of clinical characteristics of pancreatic carcinoma in northern China. Pancreas 39: 1116-1118, 2010.

2. Jemal A, Bray F, Center MM, Ferlay J, Ward E and Forman D: Global cancer statistics. CA Cancer J Clin 61: 69-90, 2011.

3. Siegel R, Naishadham D and Jemal A: Cancer statistics, 2013. CA Cancer J Clin 63: 11-30, 2013.

4. Vincent A, Herman J, Schulick R, Hruban RH and Goggins M: Pancreatic cancer. Lancet 378: 607-620, 2011.

5. Ruan K, Fang X and Ouyang G: microRNAs: novel regulators in the hallmarks of human cancer. Cancer Lett 285: 116-126, 2009.

6. Hrašovec S and Glavač D: microRNAs as Novel Biomarkers in Colorectal Cancer. Front Genet 3: 180, 2012.

7. Chen PS, Su JL and Hung MC: Dysregulation of microRNAs in cancer. J Biomed Sci 19: 90, 2012.

8. Liu H: microRNAs in breast cancer initiation and progression. Cell Mol Life Sci 69: 3587-3599, 2012.

9. Poy MN, Eliasson L, Krutzfeldt J, et al: A pancreatic isletspecific microRNA regulates insulin secretion. Nature 432: 226-230, 2004.

10. Zhang WH, Gui JH, Wang CZ, et al: The identification of miR-375 as a potential biomarker in distal gastric adenocarcinoma. Oncol Res 20: 139-147, 2012.

11. Chang Y, Yan W, He X, et al: miR-375 inhibits autophagy and reduces viability of hepatocellular carcinoma cells under hypoxic conditions. Gastroenterology 143: 177-187.8, 2012.

12. Dai X, Chiang Y, Wang Z, et al: Expression levels of microRNA-375 in colorectal carcinoma. Mol Med Rep 5: 1299-1304, 2012. 
13. Raimondi $\mathrm{C}$ and Falasca M: Targeting PDK1 in cancer. Curr Med Chem 18: 2763-2769, 2011.

14. Westmoreland JJ, Wang Q, Bouzaffour M, Baker SJ and Sosa-Pineda B: Pdk1 activity controls proliferation, survival, and growth of developing pancreatic cells. Dev Biol 334: 285-298, 2009.

15. Nikitina EG, Urazova LN and Stegny VN: microRNAs and human cancer. Exp Oncol 34: 2-8, 2012.

16. He XX, Chang Y, Meng FY, et al: microRNA-375 targets AEG-1 in hepatocellular carcinoma and suppresses liver cancer cell growth in vitro and in vivo. Oncogene 31: 3357-3369, 2012.

17. Tsukamoto Y, Nakada C, Noguchi T, et al: microRNA-375 is downregulated in gastric carcinomas and regulates cell survival by targeting PDK1 and 14-3-3zeta. Cancer Res 70: 2339-2349, 2010.

18. Shen Y, Wang P, Li Y, et al: miR-375 is upregulated in acquired paclitaxel resistance in cervical cancer. Br J Cancer 109: 92-99, 2013.

19. Garzon R, Calin GA and Croce CM: microRNAs in Cancer Annu Rev Med 60: 167-179, 2009.

20. Liu AM, Poon RT and Luk JM: microRNA-375 targets Hipposignaling effector YAP in liver cancer and inhibits tumor properties. Biochem Biophys Res Commun 394: 623-627, 2010.

21. Li X, Lin R and Li J: Epigenetic silencing of microRNA-375 regulates PDK1 expression in esophageal cancer. Dig Dis Sci 56: 2849-2856, 2011.
22. Ding L, Xu Y, Zhang W, et al: MiR-375 frequently downregulated in gastric cancer inhibits cell proliferation by targeting JAK2. Cell Res 20: 784-793, 2010.

23. Nishikawa E, Osada H, Okazaki Y, et al: miR-375 is activated by ASH1 and inhibits YAP1 in a lineage-dependent manner in lung cancer. Cancer Res 71: 6165-6173, 2011.

24. Liu Y, Xing R, Zhang X, et al: miR-375 targets the p53 gene to regulate cellular response to ionizing radiation and etoposide in gastric cancer cells. DNA Repair (Amst) 12: 741-750, 2013.

25. Sheppard K, Kinross KM, Solomon B, Pearson RB and Phillips WA: Targeting PI3 kinase/AKT/mTOR signaling in cancer. Crit Rev Oncog 17: 69-95, 2012.

26. Dhanasekaran DN and Johnson GL: MAPKs: function, regulation, role in cancer and therapeutic targeting. Oncogene 26: 3097-3099, 2007.

27. Zhang X, Yan Z, Zhang J, et al: Combination of hsa-miR-375 and hsa-miR-142-5p as a predictor for recurrence risk in gastric cancer patients following surgical resection. Ann Oncol 22: 2257-2266, 2011.

28. Song J, Kim D, Chun CH and Jin EJ: microRNA-375, a new regulator of cadherin-7, suppresses the migration of chondrogenic progenitors. Cell Signal 25: 698-706, 2013. 